Case

Report

\title{
Coronary Artery Bypass Grafting following Simultaneous Treatment of Abdominal Aortic Aneurysm and Peripheral Arterial Disease
}

\author{
Veysel Temizkan, MD, ${ }^{1}$ Murat Ugur, MD, ${ }^{1}$ Ibrahim Alp, MD,${ }^{1}$ Alper Ucak, MD, ${ }^{1}$ \\ Erturk Yedekci, MD, ${ }^{2}$ and Ahmet Turan Yilmaz, $\mathrm{MD}^{1}$
}

\begin{abstract}
Atherosclerosis might affect all arterial segments of the vascular system, thus peripheral arterial disease (PAD) accompanying coronary artery disease (CAD) is not uncommon. In addition to this coexistence, abdominal aortic aneurysm (AAA) is frequently associated with CAD. Although treatment strategies of CAD and PAD or CAD and AAA has been reported previously, treatment of these three pathologies has not been reported. The management of a therapeutic strategy is important for avoiding perioperative mortality and morbidity in CAD associated with AAA and PAD. We are reporting our simultaneous treatment strategy of three pathologies with endovascular AAA repair, stent implantation into the superficial femoral artery (SFA) and coronary artery bypass grafting (CABG).
\end{abstract}

Keywords: cardiovascular pathology, aorta and great vessels

\section{Introduction}

Abdominal aortic aneurysm (AAA) is frequently associated with coronary artery disease (CAD) especially in elderly patients. The management of a therapeutic strategy is extremely important and relevant to the severity of pathology in each clinical condition. ${ }^{1)}$ Therapeutic options are available as combined or separate operation. Nowadays, development and spread of endovascular techniques combined with surgery is a new alternative treatment technique. Following endovascular abdominal aortic aneurysm repair (EVAR), coronary artery bypass grafting $(\mathrm{CABG})$ using cardiopulmonary bypass $(\mathrm{CPB})$

${ }^{1}$ GATA Haydarpasa Training Hospital Cardiovascular Surgery Department, Istanbul, Turkey

${ }^{2}$ GATA Haydarpasa Training Hospital Anesthesiology and Reanimation Department, Istanbul, Turkey

Received: July 25, 2012; Accepted: January 28, 2013

Correspondingauthor:MuratUgur,MD.GATAHaydarpasaTraining Hospital Cardiovascular Surgery Department, Selimiye mah. Tibbiye cad. 34668, Uskudar - Istanbul, Turkey

Email: drmugur@gmail.com

(C)2014 The Editorial Committee of Annals of Thoracic and Cardiovascular Surgery. All rights reserved. can be performed. Early stent graft occlusion might develop due to insufficient runoff related to stenosis of lower limb arteries. Individual treatment strategies according to the characteristic of the lesion is important for decreasing perioperative mortality and morbidity and long term patency.

\section{Case Report}

Preoperative computed tomography and angiography evaluation of a 52-year-old morbidly obese (BMI: $40 \mathrm{~kg} / \mathrm{cm}^{2}$ ) male patient with complaints of exertional dyspnea and claudication revealed a $65.8-\mathrm{mm}$ diameter infrarenal AAA, bilateral superficial femoral artery (SFA) stenosis, $80 \%$ stenosis of the left anterior descending artery (LAD) in the proximal 1/3 segment and 70\% stenosis of the right coronary artery (RCA) main body (Fig. 1). Regarding existing symptoms and conditions, we administered a clopidogrel loading dose before the first session and undertook aorto-bi-iliac stent-graft implantation while the patient was under general anesthesia (25.5 main body, $12 \times 140 \mathrm{~mm}$ two iliac extension) (Fig. 2). Following this, bilateral lower extremity selective angiography was performed before femoral arteriotomies were closed. 


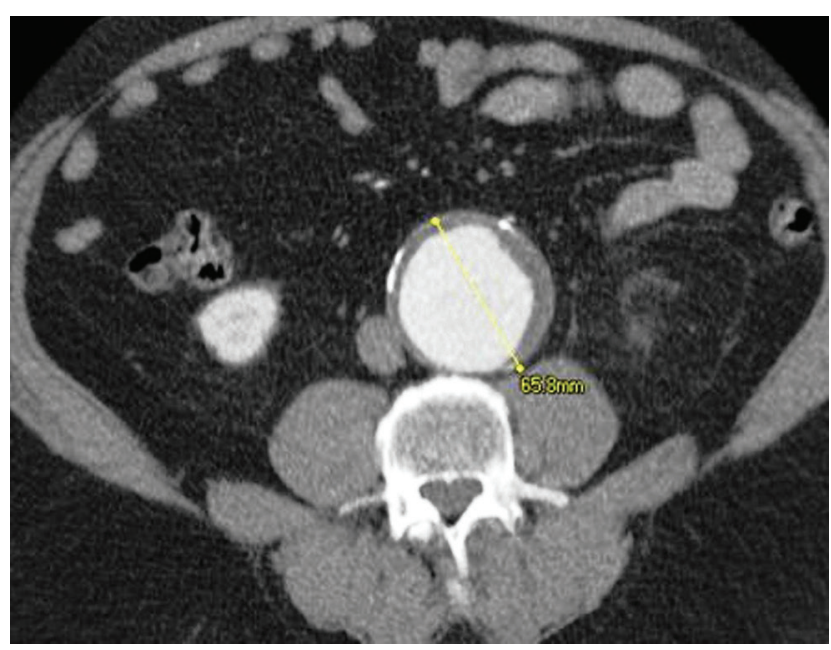

Fig. 1 Diameter of abdominal aortic aneurysm (AAA) in computed tomography.

After balloon dilatation of stenotic right SFA (50\%-60\% stenosis), $7 \times 80 \mathrm{~mm}$ stent implantation was performed in the stenotic left SFA (80\% stenosis) (Fig. 3). Postoperatively until the day of CABG clopidogrel $75 \mathrm{mg} /$ day applied, and before the coronary revascularization was initiated. On postoperative day 5 of the stent-graft implantation following median sternotomy and standard CPB, CABG was carried out for the LAD and RCA. Coronary artery bypass graft surgery was accomplished uneventfully, and the patient was transferred to the intensive care unit. He was extubated in postoperative 8th hour. Thoracic and mediastinal drainage were $250 \mathrm{mg}$ and $400 \mathrm{mg}$ respectively. The patient was discharged on the postoperative 7 th day with dual antiplatelet therapy. Postoperative evaluation of the patient's heart function after 1 month revealed no pathologic findings; peripheral arterial pulses were palpable, and preoperative symptoms had disappeared.

\section{Discussion}

Coexistence of CAD in patients with AAA is 40\%-60\% while 13\%-18\% of patients with CAD have an abdominal aorta above $3 \mathrm{~cm} .{ }^{2)}$ In terms of cases with $\mathrm{AAA}$ and $\mathrm{CAD}$ coexistence, determining the priority order is a difficult decision for both patient and surgeon. Especially in patients with an abdominal aorta diameter of over $6 \mathrm{~cm}$ and a severe LAD artery lesion, this decision is even more difficult. In two-stage treatment, if the aneurysm repair is carried out initially, cross-clamping of the abdominal aorta increases systemic vascular resistance and left ventricular wall stress, leading to subendocardial ischemia and depression of myocardial function. ${ }^{3)}$ On the other hand, aortic declamping possesses a risk for myocardial ischemia following sudden hypotension. The most important reason of perioperative mortality and morbidity following surgical treatment of AAA is CAD. ${ }^{4)}$ Patients undergoing aortic surgery are followed up between 2 weeks and 2 months after CABG. ${ }^{5)}$ When CABG was carried out first, inflammatory effects of CPB causes dilatation in AAA and decreases the tension strength applied force (tensile strength) which weakens the wall. In addition to this, increasing collagenase activity after major surgery increases the risk of rupture. ${ }^{3)}$ In cases where the priority was decided for $\mathrm{CABG}$, risk of AAA rupture during the waiting period increases up to $30 \% .^{1)}$ For reducing the risks of repeat anesthesia, length of hospitalization and hospital costs one-stage operation consisting of both CABG and AAA should be preferred. ${ }^{4)}$ A combined operation might be preferred because of the increased risk of mortality from rupture as a consequence of the waiting period of giant AAA presence. ${ }^{2,5)}$ Wolff, et al. ${ }^{5}$ ) applied one-stage surgical repair of AAA in 13 patients after $\mathrm{CABG}$, and they reported risks of perioperative mortality and morbidity as $15 \%$ and $31 \%$ respectively. To reduce the risks of perioperative mortality and morbidity of combined surgery, CABG on a beating heart is the preferred method. ${ }^{4)}$ In combined surgery performing coronary revascularization on the beating heart reduces the risk of mortality down to $0 \%$ to $5.6 \%$, but all vessels might not be revascularized in multivessel disease. ${ }^{2,4)}$

Combined surgery might be the preferred surgical procedure in symptomatic patients who are at high risk of experiencing rupture while in asymptomatic patients, AAA repair can be carried out 1 to 2 weeks after CABG. ${ }^{5)}$ With advances in endovascular techniques, an operation carried out in a single session of EVAR and CABG on a beating heart has been reported to be the optimal application and was proposed to be the preferred treatment option. ${ }^{1)}$ Effective runoff is important for stent graft patency following EVAR, as it is in the follow-up period of CABG. Early graft stenosis might be the result of poor and insufficient runoff. In our case there was no left main coronary artery (LMCA) lesion or lesions equivalent to an LMCA. His primary symptom was claudication. AAA and CAD were diagnosed during operative preparations for PAD. In morbidly obese patients undergoing abdominal surgery under emergency conditions, they might come out with high mortality and 


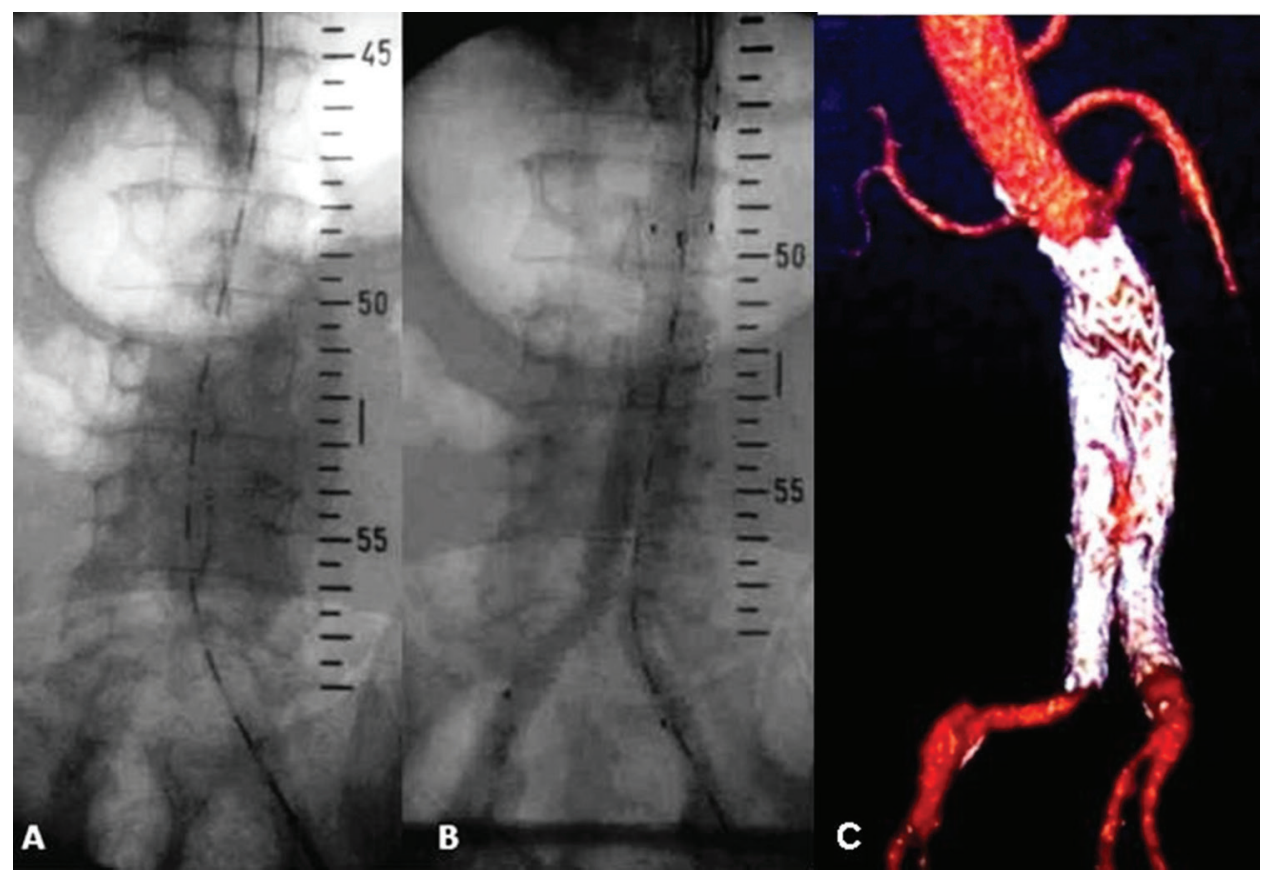

Fig. 2 (A) View of aneurysm in angiography. (B) Control angiography after stent greft implantation. (C) Postoperative computed tomographic control of abdominal aorta.

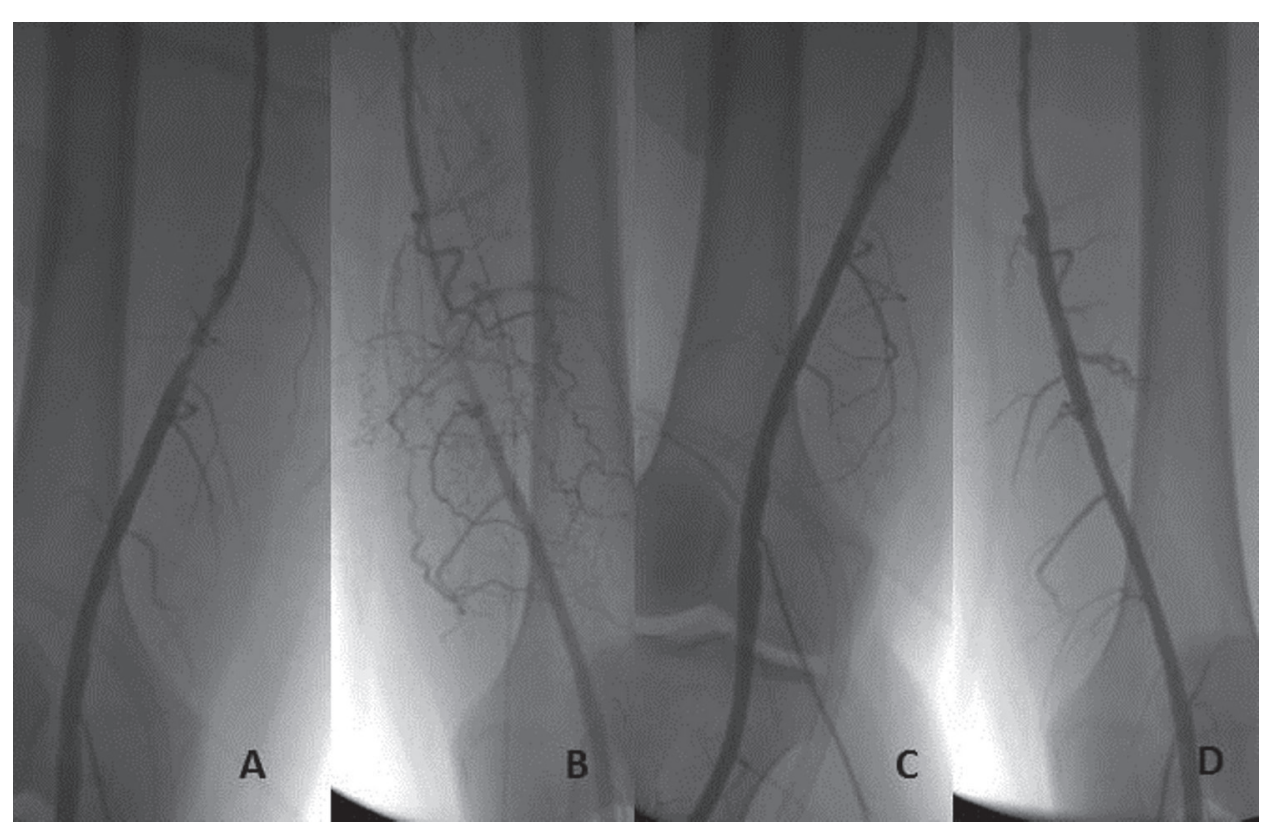

Fig. 3 (A) SFA lesion in right leg. (B) SFA lesion in left leg. (C) Control angiography after balon dilatation in right leg. (D) Control angiography of left leg after stent implantation.

morbidity. Therefore, we performed EVAR in the first stage. In this stage, if we had only treated the AAA, early stent graft thrombosis might have occurred, especially in iliac legs of the graft due to improper runoff. We believe that if there were accompanying PAD especially in iliac or femoral arteries to the aortic aneurysm, this lesion should be treated during the same period, for avoiding early term graft occlusion. For this reason, in our case following EVAR, bilateral SFA lesions were treated with endovascular interventions. Combining treatment of EVAR and peripheric arterial stenting not only avoids early term in-stent restenosis risk due to SFA lesions but 
also relieve the patients symptoms. Also treatment of peripherial arterial disease avoids restriction of mobilization after cardiac surgery. After performing the first stage and in order to prevent early stent thrombosis until CABG, we administered clopidogrel. There was no significant drainage despite the clopidogrel administration during the postoperative period. To the best of our knowledge, this is the first reported case of a patient having $\mathrm{CABG}$ following simultaneous treatment of AAA and PAD.

\section{Conclusion}

In concomitant $\mathrm{CAD}$ and $\mathrm{AAA}, \mathrm{CABG}$ can be performed after EVAR in a single session. In the presence of an LMCA lesion and huge aneurysm it is important to treat both pathologies during the same session. Revascularization of the lower limb is important for avoiding early thrombosis from the stent graft, especially of those in iliac arteries. An individual management strategy in the treatment of coexisting CAD, AAA and PAD is the main step for decreasing perioperative mortality and morbidity and reducing early term complications.

\section{Disclosure Statement}

The authors report to Annals and Thoracic and Cardiovascular Surgery that no conflict of interest exist with any companies/organizations whose products or services may be discussed in this article.

\section{References}

1) Lachat $M$, Witzke $H$, Pfammatter $T$, et al. Aortic stent-grafting: successful introduction into the combined procedure for coronary artery bypass grafting and aortic aneurysm repair. Eur J Cardiothorac Surg 2003; 23: 532-6.

2) Minami H, Mukohara N, Obo H, et al. Simultaneous operation of off pump coronary artery bypass and abdominal aortic aneurysm repair. Jpn J Thorac Cardiovasc Surg 2005; 53: 133-7.

3) Ohuchi H, Kato M, Asano H, et al. Combined coronary artery bypass grafting and abdominal aortic aneurysm repair. Asian Cardiovasc Thorac Ann 2003; 11: 233-6.

4) Morimoto K, Taniguchi I, Miyasaka S, et al. Usefulness of one-stage coronary artery bypass grafting on the beating heart and abdominal aortic aneurysm repair. Ann Thorac Cardiovasc Surg 2004; 10: 29-33.

5) Wolff T, Baykut D, Zerkowski HR, et al. Combined abdominal aortic aneurysm repair and coronary artery bypass: presentation of 13 cases and review of the literature. Ann Vasc Surg 2006; 20: 23-9. 